ARTICLE

DOI: 10.1038/s41467-017-01411-9

\title{
Anomalous elastic properties across the $\gamma$ to $\alpha$ volume collapse in cerium
}

\author{
Magnus J. Lipp ${ }^{1}$, Zs. Jenei ${ }^{1}$, H. Cynn ${ }^{1}$, Y. Kono (1) ${ }^{2}$, C. Park ${ }^{2}$, C. Kenney-Benson ${ }^{2} \&$ W.J. Evans ${ }^{1}$
}

The behavior of the f-electrons in the lanthanides and actinides governs important macroscopic properties but their pressure and temperature dependence is not fully explored. Cerium with nominally just one $4 f$ electron offers a case study with its iso-structural volume collapse from the $\gamma$-phase to the $\alpha$-phase ending in a critical point $\left(p_{C}, V_{C}, T_{C}\right)$, unique among the elements, whose mechanism remains controversial. Here, we present longitudinal $\left(c_{L}\right)$ and transverse sound speeds $\left(c_{T}\right)$ versus pressure from higher than room temperature to $T_{C}$ for the first time. While $c_{L}$ experiences a non-linear dip at the volume collapse, $c_{T}$ shows a step-like change. This produces very peculiar macroscopic properties: the minimum in the bulk modulus becomes more pronounced, the step-like increase of the shear modulus diminishes and the Poisson's ratio becomes negative-meaning that cerium becomes auxetic. At the critical point itself cerium lacks any compressive strength but offers resistance to shear.

\footnotetext{
${ }^{1}$ Lawrence Livermore National Laboratory, Livermore, CA 94550, USA. ${ }^{2}$ HPCAT, Geophysical Laboratory, Carnegie Institute of Washington, Argonne, IL 60439, USA. Correspondence and requests for materials should be addressed to M.J.L. (email: lipp1@llnl.gov)
} 
$\mathrm{n}$ the rearranged periodic table of Smith and $\mathrm{Kmetko}^{1}$, cerium takes the upper left corner sitting on the diagonal which delineates the boundary between itinerancy and localization, bonding and magnetic moment formation of the $f$-electrons. Elements sitting on this diagonal are highly susceptible to small perturbations resulting in a high number of crystallographic phases (four for cerium at atmospheric pressure), are highly reactive, or exhibit instabilities ${ }^{2}$. Other elements on this diagonal are iron and plutonium $(\mathrm{Pu})$, the latter with even more phases than cerium. The behavior of the one f-electron in $\mathrm{Ce}$ is responsible for the volume dependence under pressure which contracts abruptly by $\sim 15 \%$ at ambient temperature (RT) when the pressure reaches $\sim 0.75 \mathrm{GPa}^{3}$. This volume collapse (VC) announces itself by a gradual softening of the bulk modulus in the $\gamma$-phase which reaches a minimum at the moment of the transition and then rises again in the $\alpha$-phase with a discontinuous change in slope $e^{3-5}$. The crystallographic structure remains, unparalleled among the elements, but other than that the two fcc phases are quite different. While the $\gamma$-phase shows the magnetic susceptibility $\chi$ of a trivalent atom, the higher pressure $\alpha$-phase starts out with just one fifth of the $\gamma$-phase $\chi$-value and then loses even more ${ }^{6,7}$ hinting at tetravalency. This scenario also plays out at higher temperatures but now the transition requires more pressure and the volume change $\Delta V$ is reduced. It finally ends in a critical point at the critical pressure $P_{\mathrm{C}} \sim 1.5 \mathrm{GPa}$ and the critical temperature $T_{\mathrm{C}} \sim 480 \mathrm{~K}^{8-11}$ with $\Delta V=0$ where the isothermal bulk modulus vanishes $\left(B_{\mathrm{T}}=-V(\partial p / \partial V)_{\mathrm{T}}=0\right)$. Remnants of this behavior can be observed even beyond the critical point since $B_{\mathrm{T}}$ still exhibits a non-vanishing minimum that separates the two types of solid. Now, however, the pressure derivative $\left(\partial B_{\mathrm{T}} / \partial P\right)$ is continuous compared to the abrupt change below the critical point. With increasing temperature, the minimum becomes less pronounced, wider, and shallower ${ }^{10}$. Nevertheless, it appears to continue into the melt separating a $\gamma$-type (low density) from an $\alpha$-type (high density) liquid ${ }^{12,13}$.

This peculiar behavior has attracted a large theoretical effort-almost from day one after its discovery by Bridgman ${ }^{3}$ in 1927. Linus Pauling suggested that the VC and concomitant decrease of the magnetic susceptibility was caused by the promotion of the $f$-electron to a bond-forming orbital ${ }^{14}$. The spin-orbit coupling of the $f$-electrons in the lanthanides via Hund's rules had explained the paramagnetism of lanthanide ions in salts very well ${ }^{15}$, one of the early triumphs of quantum mechanics. It was only natural to assume that the destruction of the magnetic properties was caused by the promotion of the $f$ electron. Beyond the paramagnetism that is directly derived from the $f$-electrons in the trivalent ions, the compression behavior is also strongly affected by them but the systematics are more complex. Praseodymium-with two $f$-electrons-also suffers a volume collapse, albeit at a much higher pressure of $20 \mathrm{GPa}^{16}$, whereas neodymium's (3 f-electrons) equation of state (EOS) evolves without one ${ }^{17}$. Over the years, the promotional model of Zachariasen-Pauling gave way to many others (see ref. ${ }^{18}$ and references therein).

The current two front-runners explaining the VC mechanism are the Hubbard-Mott and the Kondo VC models ${ }^{19-21}$. The possible disappearance of the total angular momentum $J$ points to the conjecture that the localized $4 f$ electron becomes itinerant, undergoing a Mott transition ${ }^{19}$. In the other scenario, the $4 f$ electron remains localized and the VC is caused by the onset of screening of the $f$-electron magnetic moment by the conduction band electrons, the Kondo mechanism ${ }^{20,21}$. Both models attempt to quantify the $\mathrm{VC}$ by calculating the free energy and from there the EOS or phase diagram ${ }^{22,23}$. Indeed, both models can interpret the EOS above the critical point ${ }^{10,24}$ and the controversy continues.

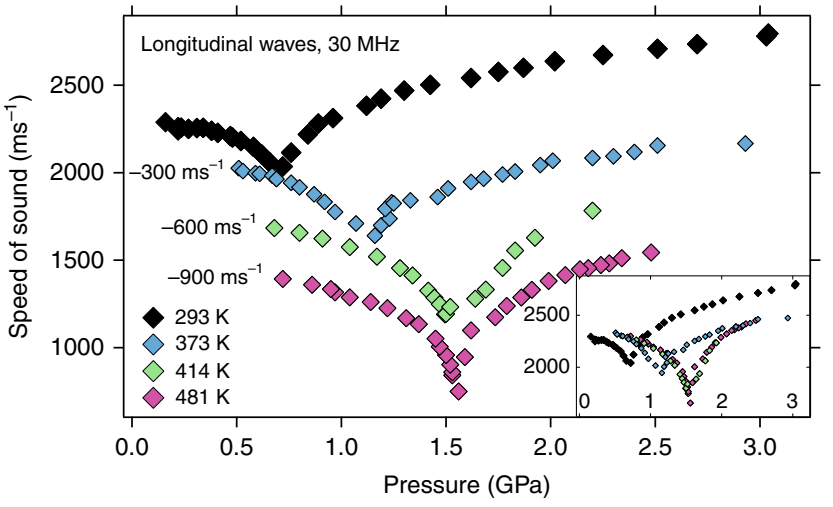

Fig. 1 Ultrasonic longitudinal velocities. The values at $293 \mathrm{~K}, 373 \mathrm{~K}, 414 \mathrm{~K}$, and $481 \mathrm{~K}$ are displayed with an offset by subtracting $300 \mathrm{~ms}^{-1}(373 \mathrm{~K})$, $600 \mathrm{~ms}^{-1}(414 \mathrm{~K})$ and $900 \mathrm{~ms}^{-1}(481 \mathrm{~K})$ for better clarity. The inset shows the same data without offsets. Uncertainty in pressure $(<0.05 \mathrm{GPa})$ and speed $(<1 \%)$ is within symbol size

Since the elastic moduli $C_{i j}$ are the second derivatives of the free energy with respect to strain and the isothermal bulk modulus $B_{\mathrm{T}}$ the volume derivative of the free energy, they offer a connection to electronic structure calculations. $B_{\mathrm{T}}$ can be found experimentally by X-ray diffraction of polycrystalline samples. The $C_{i j}$ can be studied with ultrasonic waves impinging on oriented single crystals. Measuring the transverse and longitudinal ultrasonic waves $c_{\mathrm{T}}$ and $c_{\mathrm{L}}$ in polycrystalline samples, one can obtain the pressure dependence of the adiabatic bulk modulus $B_{\mathrm{S}}$ and the shear modulus $G^{5,25-28}$. Previous studies at RT also derived the Debye temperatures $\theta_{\gamma}$ and $\theta_{\alpha}$ in each phase $e^{5,27,28}$. A significant part of the total entropy change $\Delta S$ across the volume collapse given by the Clausius-Clapeyron relation $\Delta S=$ $\Delta V \cdot \mathrm{d} P / \mathrm{d} T$ apparently originates with the lattice contribution $\Delta S_{\mathrm{vib}}=3 k_{\mathrm{B}} \cdot \ln \left(\theta_{\gamma} / \theta_{\alpha}\right)^{4,5,25-28}$. The actual size is still under debate theoretically $29-31$ and experimentally $4,5,26-28,32 . \Delta S$ is almost equal to $\Delta S_{\mathrm{J}}=k_{\mathrm{B}} \ln \left[\left(2 J_{\gamma}+1\right) /\left(2 J_{\alpha}+1\right)\right]$ with $J_{\gamma}=5 / 2$ and possibly $J_{\alpha}=0$ as the Hund's rule moments in their respective phases. The assumption $J_{\alpha}=0$ is very appealing since it explains the $80 \%$ drop of the magnetic susceptibility across the $\mathrm{VC}^{6,7}$ but it does not leave room for a sizable lattice contribution.

Here, we present measurements of the transverse and longitudinal sound speeds in polycrystalline cerium at higher temperatures than ambient. We find that the step-like increase in $G$ at the VC decreases with temperature and disappears, and-for temperatures approaching the critical point-a negative Poisson's ratio which makes elemental cerium auxetic under these conditions. The decrease in the bulk modulus $B_{\mathrm{S}}$ approaching the VC appears to be caused solely by the decrease in the elastic constant $C_{12}$. The lattice contribution $\Delta S_{\text {vib }}$ to the total entropy change $\Delta S$ becomes less important with temperature while both vanish at the critical point just like the intrinsic hysteresis. Other mechanisms must then fill the widening gap in $\Delta S$, such as increasing thermal disorder.

\section{Results}

Ultrasonic velocities. Cerium shows some unexpected behavior of the longitudinal and transverse velocities $c_{\mathrm{L}}$ and $c_{\mathrm{T}}$ with pressure. The values for the ultrasonic velocities are displayed in Figs. 1 and 2 for temperatures of 293, 373, 414, and $481 \mathrm{~K}$, with $481 \mathrm{~K}$ very close to or at the critical temperature ${ }^{8-11}$

Looking at the 373,414 , and $481 \mathrm{~K}$ isotherms in Fig. 1 for the longitudinal sound speeds one finds that they all start out at $\sim 2300 \mathrm{~ms}^{-1}$ when the pressures are far from the volume collapse pressure $P_{\mathrm{VC}}$ which increases with temperature ${ }^{18}$. At higher 


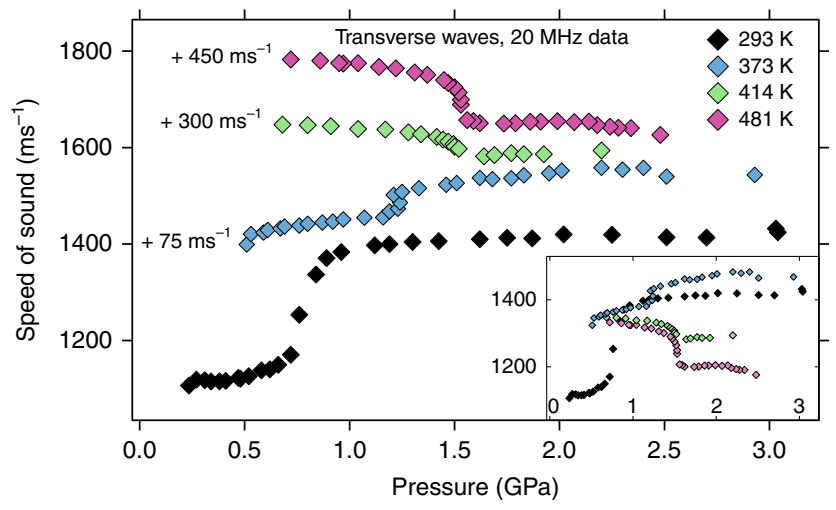

Fig. 2 Ultrasonic transverse velocities. The values at $293 \mathrm{~K}, 373 \mathrm{~K}, 414 \mathrm{~K}$, and $481 \mathrm{~K}$ are displayed with an offset by adding $+75 \mathrm{~ms}^{-1}(373 \mathrm{~K})$, $+300 \mathrm{~ms}^{-1}(414 \mathrm{~K})$, and $+450 \mathrm{~ms}^{-1}(481 \mathrm{~K})$ for better clarity. The inset shows the same data without offsets. Uncertainty in pressure $(<0.05 \mathrm{GPa})$ and speed $(<1 \%)$ is within symbol size

temperature, $c_{L}$ decreases even more approaching the transition pressure, at $481 \mathrm{~K}$ down to $1600 \mathrm{~ms}^{-1}$. After the VC in the $\alpha$ phase, all wave speeds increase again about as quickly as they decreased.

Figure 2 shows the completely different behavior of the transverse waves. They either climb (373 K) or decrease slightly (414 and $481 \mathrm{~K}$ ) with pressure far away from the transition in each phase but show a pronounced increase $(373 \mathrm{~K})$ or decrease (414 and $481 \mathrm{~K}$ ) at the transition pressure. Also, at $373 \mathrm{~K}$, the transverse wave speed in the $\gamma$-phase has already exceeded the transverse wave speeds observed at RT previously by others $5,25,26,28$ but does not change much further with temperature up to $481 \mathrm{~K}$.

A detailed comparison to RT data of other authors has been published previously ${ }^{27}$. While all the RT data for the longitudinal sound speed are in very good agreement in the $\gamma$-phase, the values for the transverse wave speeds are somewhat different with the ones in this study being $\sim 200 \mathrm{~ms}^{-1}$ lower. Beyond the VC the transverse values are again in agreement ${ }^{25-27}$, which indicates that the present sample environment ${ }^{28}$ provides trustworthy data. The possible reason for the transverse sound speed difference in the $\gamma$-phase has been discussed at length in ref. ${ }^{27}$ before and is most likely caused by different sample treatment. The samples used in the present study have not been subjected to long periods of heating ${ }^{5}$ or even melting and subsequent quenching ${ }^{26}$. In fact, our values for the transverse speed lead to a much closer agreement with previously published Debye temperature data obtained by measurements of the complete phonon dispersion relation in cerium under pressure by either inelastic neutron or $\mathrm{X}$-ray scattering on single crystals ${ }^{27}, 32,33$.

Adiabatic bulk modulus $\boldsymbol{B}_{\mathrm{S}}$ and shear modulus $\boldsymbol{G}$. With the previously measured densities for the chosen temperatures ${ }^{10}$ we are able to obtain values for the shear modulus $G=\rho c_{\mathrm{T}}{ }^{2}$ and adiabatic bulk modulus $B_{\mathrm{S}}=\rho\left(c_{\mathrm{L}}^{2}-4 / 3 c_{\mathrm{T}}^{2}\right)$ under pressure (Fig. 3a). The minimum of the bulk modulus $B_{\mathrm{S}}$ at the VC at RT continues at higher temperatures with a deeper and steeper minimum. As for the shear modulus $G$, cerium shows an initial increase in the $\gamma$-phase with temperature-contrary to the usually observed decrease in metals. Under pressure, $G$ rises slightly in the $\gamma$-phase and then increases step-like across the VC from the $\gamma$-phase to the $\alpha$-phase but this step vanishes with temperatures closer to $T_{\mathrm{C}}$. At the critical point $\mathrm{Ce}$ becomes a solid without compressive strength $\left(B_{\mathrm{T}}=0\right)$ but finite shear strength.
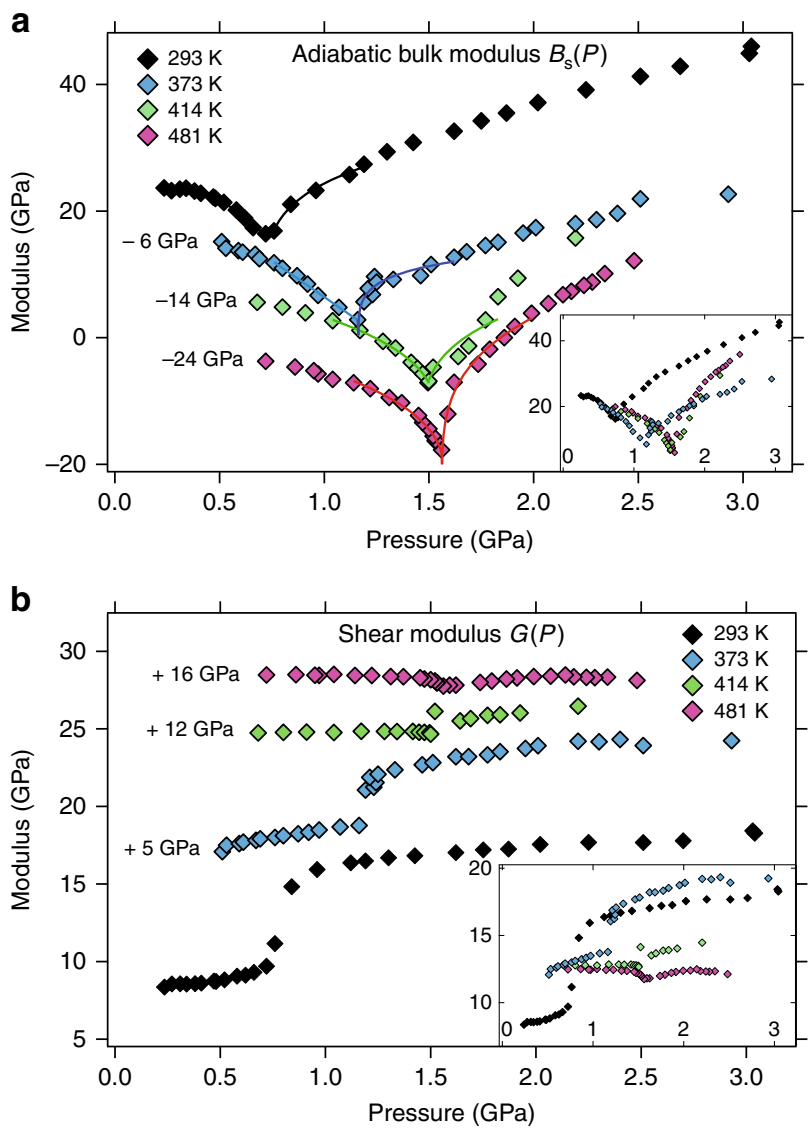

Fig. 3 Pressure dependence of the adiabatic bulk and shear moduli. a Values for the adiabatic bulk modulus $B_{\mathrm{S}}=\rho\left(c_{\mathrm{L}}{ }^{2}-4 / 3 c_{\mathrm{T}}{ }^{2}\right)$ are shown for different isotherms. Width and depth of the transition for $B_{S}$ become narrower and deeper with the temperature approaching the critical temperature $T_{\mathrm{C}}$. A similar behavior has been observed for the isothermal bulk modulus $B_{\mathrm{T}}$ above $T_{\mathrm{C}}$ where the width of the transition becomes wider and the minimum shallower with further distance from $T_{\mathrm{C}}$. At $T_{\mathrm{C}}$ itself $B_{\mathrm{T}}$ vanishes at the critical pressure $P_{C}{ }^{10,11}$. The solid lines are the fits of a power law $B_{\mathrm{S}}(P) \sim\left|P-P_{\vee C}\right|^{\alpha}$ to the data. $\mathbf{b}$ Values for the shear modulus $G=$ $\rho c_{\mathrm{T}}^{2}$ at different temperatures. Uncertainty in pressure $(<0.05 \mathrm{GPa})$ and modulus $(<3 \%)$ is within symbol size

The adiabatic bulk modulus decreases in the $\gamma$-phase for all temperatures until it reaches its minimum at the VC transition and starts rising again continuously in the $\alpha$-phase. With higher temperature, the minimum moves to a higher pressure and becomes deeper. Since $B_{\mathrm{T}}=0$ at the critical point ${ }^{10,11}$ and $B_{\mathrm{S}}=$ $B_{\mathrm{T}} C_{\mathrm{P}} / C_{\mathrm{V}}$ it follows that $B_{\mathrm{S}}$ should fall to zero at the critical point just as well since $C_{\mathrm{P}}$ and $C_{\mathrm{V}}$ are nonzero ${ }^{34,35}$. Our lowest observed value is $B_{\mathrm{S}} \sim 6 \mathrm{GPa}$ for $p=1.55 \mathrm{GPa}$ at $481 \mathrm{~K}$ which could mean that our temperature was just barely different from the critical temperature. It is possible to fit the pressure dependence of $B_{\mathrm{S}}$ near the transition to a power law $B_{\mathrm{S}}(P) \sim \mid P$ $-P_{\mathrm{VC}}{ }^{\alpha 4,26}$. The solid lines in Fig. 3a show the resulting fits to the data. The values for $P_{\mathrm{VC}}$ and $\alpha$ are listed in Table 1 for a pressure range of about $0.4 \mathrm{GPa}$ below (-) and above (+) the transition. However, except for the critical temperature, the transition already takes place at $P<P_{\mathrm{VC}}$. This was observed previously by X-ray diffraction for $B_{\mathrm{T}}{ }^{4}$ and ultrasonic measurements for $B_{\mathrm{S}}{ }^{26}$ at room temperature which found $\alpha^{-}=0.46, P_{\mathrm{VC}}{ }^{-}=0.83 \mathrm{GPa}^{4}$ and $\alpha^{-}=0.42, P_{\mathrm{VC}}{ }^{-}=0.92 \mathrm{GPa}^{26}$, respectively. At the critical temperature $(481 \mathrm{~K})$ we find $\alpha^{-}=0.26$ and $\alpha^{+}=0.28$, basically equal within the experimental uncertainty. An exponent of 0.5 would indicate Gaussian fluctuations ${ }^{4}$ associated with electron-phonon 
Table 1 Pressure $P_{\mathrm{vc}}(\mathrm{GPa})$ and exponent $\alpha$ obtained by fitting $B_{\mathrm{s}}(P) \sim\left|P-P_{\mathrm{vc}}\right|^{\alpha}$ to the data

\begin{tabular}{llll}
$\mathbf{T}(\mathbf{K})$ & $\boldsymbol{P}_{\mathbf{v c}}{ }^{-}, \boldsymbol{P}_{\mathbf{v c}}{ }^{+} \mathbf{( G P a )}$ & $\boldsymbol{\alpha}^{-}, \boldsymbol{\alpha}^{+}$ & Method \\
\hline 289 & $0.89(3), 0.49(4) \Delta P=0.4$ & $0.27(2), 0.11(3)$ & (a) Ultrasound \\
293 & $0.79(5), 0.61(16) \Delta P=0.18$ & $0.35(7), 0.3(4)$ & (b) Ultrasound \\
RT & $0.83,-$ & $0.46,-$ & (c) X-ray diffraction \\
RT & $0.92,-$ & $0.42,-$ & (d) Ultrasound \\
293 & $0.89(11), 0.71(3) \Delta P=0.18$ & $0.34(13), 0.20(5)$ & Present work, ultrasound \\
373 & $1.25(11), 1.16(1) \Delta P=0.09$ & $0.50(17), 0.14(2)$ & Present work, ultrasound \\
414 & $1.53(1), 1.47(4) \Delta P=0.06$ & $0.34(2), 0.32(8)$ & Present work, ultrasound \\
481 & $1.55(1), 1.56(1) \Delta P=0.01$ & $0.26(2), 0.28(3)$ & Present work, ultrasound \\
\hline
\end{tabular}

The superscripts - and + refer to the results for the low $(-)$ and high pressure $(+)$ fitting range (a) ref. ${ }^{5}$ (b) ref. ${ }^{25}$ (c) ref. ${ }^{4}$ (d) ref. ${ }^{26}$

a Fit to previously published data performed within present work

coupling $^{26}$, an exponent of zero is expected in the mean-field theory. Our value of $\alpha=0.27 \pm 0.01$ is the only one taken approximately on the critical isotherm which allows a straight forward interpretation. All other values were obtained at lower temperatures for which the descent of the bulk modulus towards zero is interrupted by the transition and thus the measured exponents are not truly "critical"4. Additionally, the further the temperature is from $T_{\mathrm{C}}$ the larger is the gap in the transition pressures $P_{\mathrm{VC}}{ }^{-}$and $P_{\mathrm{VC}}{ }^{+}$which we list in Table 1 as $\Delta P$. This value can serve as a measure of hysteresis that disappears approaching the critical temperature. Table 1 also provides a comparison with previously published results and includes values for fits to the data of refs. ${ }^{5,25}$ that were obtained within the current effort. With the exception of the $481 \mathrm{~K}$ isotherm, all exponents measured on the lower pressure side are larger than the ones from the higher-pressure side which one would expect similarly for the behavior of a van der Waals liquid/gas near the critical point.

Based on their RT data Jeong et al. ${ }^{4}$ stated that the drop in $B_{\mathrm{T}}$ towards the $\mathrm{VC}$ follows directly from the decrease of the $C_{11}$ elastic constant since $B=1 / 3\left[C_{11}+2 C_{12}\right]=1 / 3\left[3 C_{11}-4 C^{\star}\right]$ for a cubic lattice and the shear modulus $C^{\star}=\left(C_{11}-C_{12}\right) / 2$ would be insensitive to pressure ${ }^{5}$. This assumption, however, is not warranted since $C^{\star}$ is just one of the principal shear moduli, the other one being $C_{44}$. The behavior within each phase of the relatively pressure-insensitive shear modulus $G$ (Fig. 3b) for polycrystalline cerium in the Voigt or Reuss limit is dominated by $C_{44}$ since $C_{44}$ is unusually large. $C_{44}(19.4 \mathrm{GPa}$ at ambient pressure) amounts to $67-80 \%$ of the value of $C_{11}{ }^{33,36}$, a property not found to that extent in any other element except $\delta-\mathrm{Pu}^{33}, 37$. Indeed, at $p=0.8 \mathrm{GPa}, C_{44}>C_{11}{ }^{32}$. Moreover, Krisch et al. state that the phonon dispersion relations in general do not evolve strongly under compression in the $\gamma$-phase but change significantly at the location of the $\gamma-\alpha$ transition $^{32}$. Close inspection of the phonon dispersion relations of cerium under pressure published by them ${ }^{32}$ shows that $C_{11}$ does not decrease under compression. This leaves only $C_{12}$ as the elastic constant responsible for the pressure dependence of $B=1 / 3\left(C_{11}+2 C_{12}\right)$. $C_{12}$ is already much smaller than $C_{44}$ at ambient conditions (Stassis et al. ${ }^{33}$ report $C_{12}=10.2 \mathrm{GPa}$ for $\gamma$-Ce at RT) and its decrease therefore must be the only source for the pressure dependence of $B . C_{12}$ does not participate in waves propagating in the [100] direction, but affects the wave speed in the [110] and [111] direction, in expressions that also contain $C_{11}$ and/or $C_{44}{ }^{38}$, consistent with the highly directional character of the $f$-orbital bonding. At RT, $B$ does not drop all the way to zero since the acoustic phonon frequencies do not soften.

Additionally, at RT, $C_{12}$ itself does not vanish under pressure since in that case $B_{\mathrm{S}}$ would drop to $\sim 7 \mathrm{GPa}$ which is clearly not seen in our or others' experiments ${ }^{5,26,28}$. In principle, however, a negative $C_{12}$ would not be prohibited as has been observed for intermediate valent compounds of $4 f$ metals like TmSe or $\mathrm{Sm}_{x} \mathrm{La}_{1-x} \mathrm{~S}^{39,40}$. While small, the effect of $C_{12}$ on bulk and shear modulus is not negligible. Since the bulk modulus is the same for the Voigt and Reuss limits, $B=B_{\mathrm{V}}=B_{\mathrm{R}}=1 / 3\left(C_{11}+2 C_{12}\right)=0$ at the critical point $(\sim 1.5 \mathrm{GPa}$ and $480 \mathrm{~K})$, it follows that $C_{12}=-1 / 2$ $C_{11}$. At the critical point $C_{12}$ is indeed negative and exactly half the size of $C_{11}$.

$C_{12}$ enters both shear modulus limits: $G_{\mathrm{V}}=1 / 5\left(C_{11}-C_{12}+\right.$ $\left.3 C_{44}\right)$ in the Voigt limit assuming constant strain and in the Reuss limit assuming constant stress $G_{\mathrm{R}}=5\left(C_{11}-C_{12}\right) C_{44} /\left[4 C_{44}+3\right.$ $\left(C_{11}-C_{12}\right)$. This can be simplified even more since $C_{44}$ becomes larger than $C_{11}$ at a pressure of $0.8 \mathrm{GPa}^{32}$ at RT and therefore both must be approximately equal at the pressure of the volume collapse. If we make this assumption that $C_{44} \approx C_{11}=-2 C_{12}=C$ at the critical point, we can simplify the expressions for $G_{\mathrm{V}}$ and $G_{\mathrm{R}}$ to $G_{\mathrm{V}}=0.9 C$ and $G_{\mathrm{R}}=15 / 17 C \approx 0.88 C$. The real $G$ should fall between the very narrow gap between $G_{\mathrm{V}}$ and $G_{\mathrm{R}}$. $G$ is finite and non-vanishing. Under the same assumptions the anisotropy ratio $A=C_{44} / 1 / 2\left(C_{11}-C_{12}\right)$, which is 2.8 at ambient conditions, decreases to about $A \approx 4 / 3$ at the critical point.

The pressure insensitivity of the acoustic phonons in Ce was already anticipated by Entel and Grewe ${ }^{41}$ in the context of their enhanced periodic Anderson model for cerium which was confirmed experimentally in X-ray scattering experiments ${ }^{32}$. Instead Entel et al. expected a softening of the longitudinal optical phonons when approaching the phase boundary ${ }^{41}$ which was not detected.

Figure $3 \mathrm{~b}$ shows the remarkable behavior of the shear modulus $G=\rho c_{\mathrm{T}}^{2}$. Increasing the temperature to $373 \mathrm{~K}$ also increases $G$ in both phases compared to $293 \mathrm{~K}$ but the step-like increase at the transition now amounts to only about half of the step-like transition at $293 \mathrm{~K}$. Within both phases $G$ rises slightly by about $2 \mathrm{GPa} / \mathrm{GPa}$. At $414 \mathrm{~K}$ the step at the transition becomes even smaller (about $1 \mathrm{GPa}$ ) and for $481 \mathrm{~K}$ the step has essentially vanished with the exception of a small dip at a pressure of $1.56 \mathrm{GPa}$. At $414 \mathrm{~K}$ there is almost no change under pressure within each phase and the data at $481 \mathrm{~K}$ show a very slight decrease in $G$ with pressure. At $\sim 0.8 \mathrm{GPa}$ pressure the shear modulus remains basically constant for all temperatures at $\sim 12.5 \mathrm{GPa}$. In contrast, the behavior of a normal metal would have $G$ slightly increasing with pressure and decreasing with temperature.

We established above-under the assumption that $C_{44} \approx C_{11}$ at the critical point $-\mathrm{G}_{\mathrm{V}}=0.9 \mathrm{C}$ and $G_{\mathrm{R}} \approx 0.88 C$. Since $G=12 \mathrm{GPa}$ under these conditions it follows that $C_{44} \approx C_{11} \approx 13.5 \mathrm{GPa}$ and $C_{12} \approx-6.8 \mathrm{GPa}$.

The Poisson's ratio $\nu$. The ratio of $B / G$ determines the material response of transverse strain $\delta \varepsilon_{\mathrm{T}}$ to longitudinal strain $\delta \varepsilon_{\mathrm{L}}$ via the Poisson's ratio $\nu=-\delta \varepsilon_{\mathrm{T}} / \delta \varepsilon_{\mathrm{L}}=(3 B-2 G) /(6 B+2 G)$. The Poisson's 

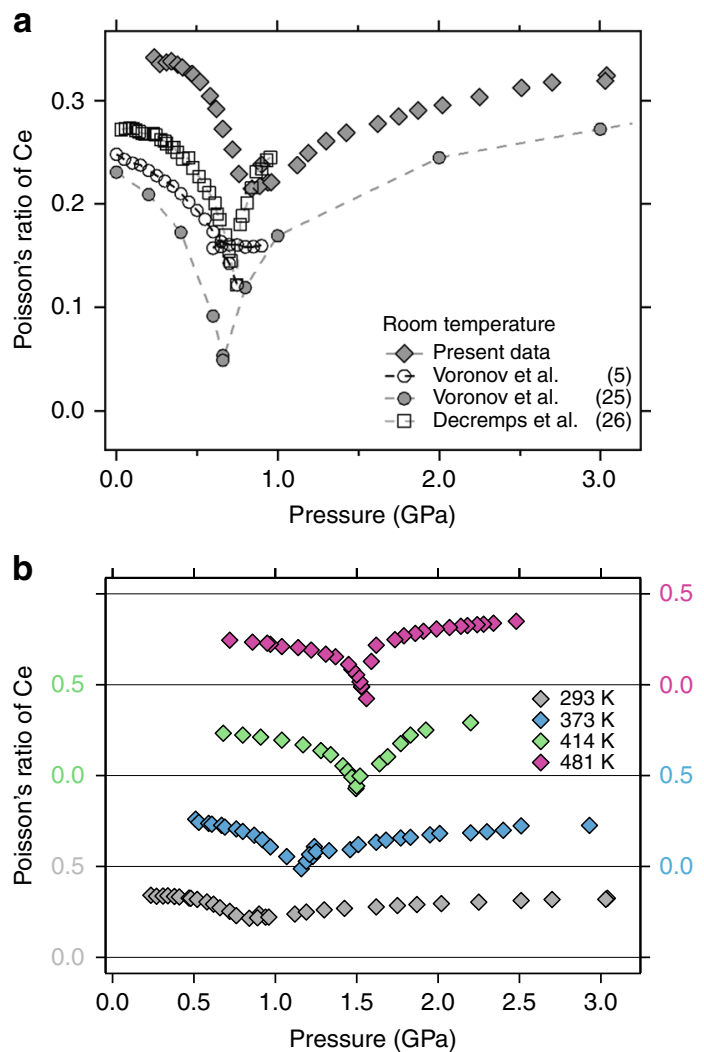

Fig. 4 The Poisson's ratio $\nu=(3 B-2 G) /(6 B+2 G)$ versus pressure. a Comparison of ambient temperature values. Values from refs. 25,26 are calculated from their published results for $B$ and $G$. b The Poisson's ratio versus pressure for different temperatures. The horizontal lines represent values of 0 and 0.5 . Towards $T_{C}$ the width of the transition decreases and the minimum becomes deeper and negative. Uncertainty in pressure is $<0.05 \mathrm{GPa}$ and the symbols for the present data are larger than the error bars

ratio becomes 0.5 for liquids $(G=0)$ which serves as a diagnostic for melting in shock wave experiments of cerium, iron and others $^{42-44}$. A comparison of Poisson's ratios at RT with previously published results is shown in Fig. 4a. While the absolute values from different authors show a substantial spread in the $\gamma$ phase they all exhibit qualitatively the same features: a slight decrease with pressure is followed by a significant drop towards the VC. In the $\alpha$-phase the Poisson's ratios rise again. In the case of Voronov's data from $1979^{25} \nu$ drops to 0.05 at the VC.

Interestingly, $\nu$ can become negative for the right ratios of $B$ and $G$, a feature that has been sought to exploit in engineering applications of other such materials ${ }^{44,45}$. This turns out to be the case for cerium at the VC at higher temperatures closer to the critical point (Fig. 4b). For our temperatures of 373,414 , and $481 \mathrm{~K}$ (Fig. 4b), all the data show a drop of $\nu$ below 0 near the VC. This drop becomes deeper and steeper with increasing temperature. Since $B_{\mathrm{S}}$ should be zero at the critical point the Poisson's ratio should drop down to -1 for $\left(P_{\mathrm{C}}, T_{\mathrm{C}}\right)$. Our lowest observed value is $\sim-0.1$ at $p=1.56 \mathrm{GPa}$. In other words, close to the $\mathrm{VC}$, cerium metal becomes auxetic approaching the critical temperature, meaning an axial compression would result in a lateral contraction (or an axial elongation results in a lateral widening).

Cerium is unique in this regard, since the reason lies with the behavior of the $4 f$ electron configuration. Typically, auxetic materials have open structures like foams ${ }^{46}$ or $\alpha$-cristobalite, a phase of $\mathrm{SiO}_{2}{ }^{47}$. Once considered an exotic and rare characteristic of materials it has been found that at least in certain specific

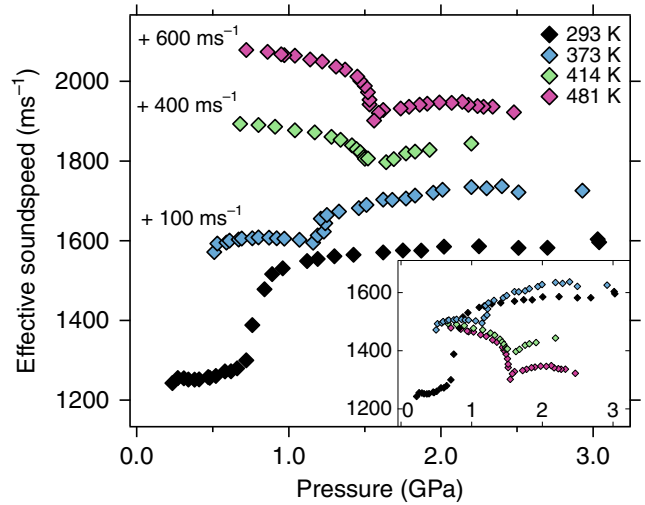

Fig. 5 Effective sound speed in cerium. The effective sound speed is a measure of the stiffness of the crystal ${ }^{15}$ and is shown versus pressure (uncertainty < $0.05 \mathrm{GPa}$ ) at $293 \mathrm{~K}, 373 \mathrm{~K}, 414 \mathrm{~K}$, and $481 \mathrm{~K}$. Curves individually offset for clarity. Symbols are larger than the experimental uncertainties

crystallographic directions such as the [110], many cubic metals exhibit a negative Poisson's ratio ${ }^{45}$. For cerium however, at elevated temperatures near the $\mathrm{VC}$, there are no restrictions with regard to crystallographic directions and no open structures are required for one of the participating phases.

Traversing the transition from the $\alpha$-phase to the $\gamma$-phase by temperature instead of pressure should show a very similar behavior of $\nu$-a decrease towards the VC, possibly to negative values, followed by an increase back to similar values as they were before the VC. This behavior due to the $f$-electron configuration in cerium is therefore quite different from that of the $5 f$ metal $\mathrm{Pu}$. The temperature dependence of the Poisson's ratio in Pu shows a large discontinuous step-like increase ( $\alpha-\beta$ phase transition) and then a decrease ( $\beta-\gamma$ phase transition) - over a temperature range of just $100 \mathrm{~K}$ as if the different phases were completely different metals ${ }^{48}$. Theoretically this has been attributed to an increase in $f$-electron correlation ${ }^{49}$. In cerium at ambient temperature the $\mathrm{f}$ electron occupancy experiences a $\sim 20-30 \%$ drop at the $\mathrm{VC}^{50,51}$. The aforementioned cubic intermediate valence compounds $\mathrm{Sm}_{x} \mathrm{La}_{1-x} \mathrm{~S}$ and TmSe also exhibit a negative Poisson's ratio which is due to a negative $C_{12}$ elastic constant ${ }^{39,40}$.

Debye temperature and lattice contribution. The knowledge of $c_{\mathrm{L}}$ and $c_{\mathrm{T}}$ also allows us to estimate the lattice contribution to the total entropy change across the $\mathrm{VC}$ for different temperatures. As it turns out the lattice contribution vanishes faster than the total entropy itself suggesting that other mechanisms need to come into play to stabilize the $\gamma$-phase against the $\alpha$-phase. Increasing thermal disorder could be a candidate.

Figure 5 displays the effective sound speed which is needed for the calculation of the Debye temperature $\theta_{\mathrm{D}}(p)$. Knowledge of the change of the Debye temperature across the VC allows a determination of the lattice contribution to the total entropy change. $C_{\text {eff }}$ is obtained from $c_{\mathrm{T}}$ and $c_{\mathrm{L}}$ according to (e.g., ref. ${ }^{52}$ ):

$$
C_{\text {eff }}=\left(\frac{1}{3}\left(\frac{2}{c_{\mathrm{T}}^{3}}+\frac{1}{c_{\mathrm{L}}^{3}}\right)\right)^{-\frac{1}{3}}
$$

$C_{\text {eff }}$ offers an assessment of the stiffness of the crystal ${ }^{15}$. At $293 \mathrm{~K}$ and $373 \mathrm{~K}$ it is lower in the $\gamma$-phase than in the $\alpha$-phase but this behavior reverses for 414 and $481 \mathrm{~K}$. At $293 \mathrm{~K}$ the increase amounts to more than $25 \%$ but reduces to $<5 \%$ at $373 \mathrm{~K}$. At $414 \mathrm{~K} C_{\text {eff }}$ drops from the $\gamma$-phase to the $\alpha$-phase by $\sim 4 \%$ and by almost $10 \%$ at $481 \mathrm{~K}$. The effective sound speed enters into the 


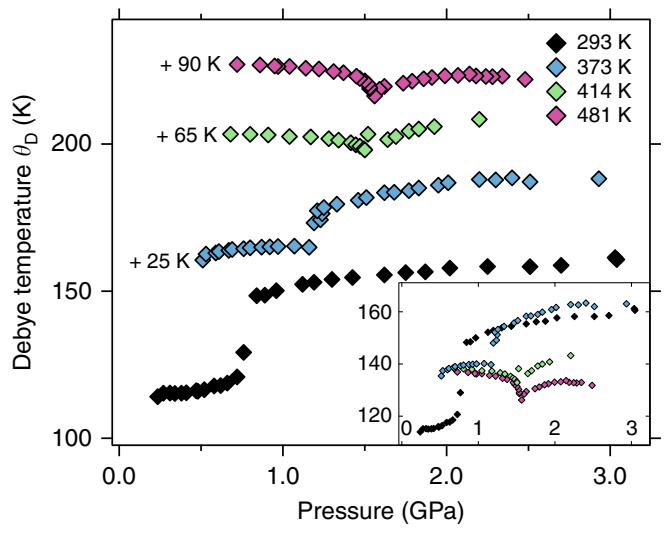

Fig. 6 Debye temperatures of polycrystalline cerium. They are shown versus pressure (uncertainty $<0.05 \mathrm{GPa}$ ) at different temperatures. Symbols are larger than the experimental uncertainties

determination of the Debye temperature via:

$$
\theta_{\mathrm{D}}=\frac{h}{2 k_{\mathrm{B}}} \cdot C_{\mathrm{eff}} \cdot \sqrt[3]{\frac{6}{\pi V_{\mathrm{at}}}} \sim \frac{C_{\mathrm{eff}}}{a}
$$

with $h$ as Planck's constant, $k_{\mathrm{B}}$ as the Boltzmann constant, $V_{\text {at }}$ the atomic volume of a cerium atom and $a$ as the lattice constant ${ }^{52}$. The change in the lattice constant going from the $\gamma$-phase to the $\alpha$-phase is $5.1 \%$ at $293 \mathrm{~K}$ and less at higher temperatures.

Figure 6 shows the Debye temperatures $\theta_{\mathrm{D}}$ derived this way. At high temperatures these can be used to approximate the lattice contribution $\Delta S_{\text {vib }}=3 k_{\mathrm{B}} \ln \left(\theta_{\alpha} / \theta_{\gamma}\right)$ to the total entropy change across the VC transition $\Delta S=\Delta V \mathrm{~d} P / \mathrm{dT}^{4}$. We used $\mathrm{d} P / \mathrm{d} T=$ $5.14 \times 10^{6} \mathrm{Jm}^{-3} \mathrm{~K}^{-1} 27$. Table 2 lists all the involved quantities.

The last column of Table 2 shows that the contribution $\Delta S_{\text {vib }}$ to the total entropy change becomes less important for higher temperatures. While it still supplies almost $40 \%$ at RT, that share drops to $<20 \%$ at $373 \mathrm{~K}$ and to $<10 \%$ at $414 \mathrm{~K}$. Since there is general consensus that the $\gamma-\alpha$ transition is entropy driven $^{29-31}, 53,54$, another mechanism has to fill the growing gap in entropy and acquire more weight with temperature. One natural candidate is the increasing amount of thermal disorder. Indeed, Jarlborg ${ }^{55}$ has found via DFT calculations on large supercells that this disorder produces fluctuations in the magnitude of the magnetic moments leading to an increase in the average moment for the low pressure $\gamma$-phase. The resulting entropic contribution additionally stabilizes the $\gamma$-phase at high temperatures with respect to the $\alpha$-phase ${ }^{55}$.

\section{Discussion}

The VC phase transition in Ce starts with the nucleation and growth of many independent crystallites in the $\alpha$-phase uniformly dispersed in the $\gamma$-phase based on the observation that a small diameter X-ray beam of $20 \mu \mathrm{m}$ always detects both phases once the transformation starts ${ }^{56}$. The transformed regions remain in the same crystallographic orientation as the non-transformed material. This allows, for example, for a single crystal in the $\gamma$ phase to become a single crystal in the $\alpha$-phase while dislocations and deformation bands are also created ${ }^{56}$. The ultrasonic waves in the current experiment probe regions that are even larger and are not sensitive to details on the atomic but on the macroscopic ( = averaged) scale. Indeed, due to the iso-structural nature of the collapse, the step-like change in the transverse velocity and shear modulus can be obtained by simply averaging the individual properties of the two distinct phases in the mixed-phase region according to their concentration.

\begin{tabular}{|c|c|c|c|c|c|c|}
\hline$T(K)$ & $\Delta V\left(\AA^{3}\right)$ & $\Delta S$ in $k_{B}$ & $\theta_{\gamma}(K)$ & $\theta_{\alpha}(K)$ & $\Delta S_{\text {vib }}$ in $k_{B}$ & $\begin{array}{l}\text { Ratio } \\
\Delta \boldsymbol{S}_{\mathrm{vib}} / \Delta \boldsymbol{S}\end{array}$ \\
\hline 293 & 4.74 & 1.765 & 119 & 149 & 0.67 & 0.38 \\
\hline 373 & 4.04 & 1.505 & 140 & 153 & 0.27 & 0.18 \\
\hline 414 & 3.64 & 1.356 & 133 & 138 & 0.11 & 0.08 \\
\hline 481 & 0 & 0 & \multicolumn{2}{|c|}{$\begin{array}{l}\text { No discernible } \\
\text { step }\end{array}$} & 0 & - \\
\hline
\end{tabular}

Many of the current results are consistent with a non-linear elastic model of a discontinuous volume change advanced by Bustingorry et $\mathrm{al}^{57}$. Under the assumption of a coherent transformation (i.e., without taking structural defects into account) between two isotropic phases with the same bulk and shear modulus it is expected that the bulk modulus softens according to $B \sim\left|P-P_{\mathrm{Vc}}\right|^{\alpha}$ with $\alpha=1 / 2$ even at temperatures far away from the critical point. While our measured exponents are different from $1 / 2$ (Table 1 ), a softening of the bulk modulus according to a power law is clearly observed. This also implies that the Poisson's ratio becomes negative ${ }^{57}$, again experimentally observed by us.

The difference between $P_{\mathrm{VC}}{ }^{-}$and $P_{\mathrm{VC}}{ }^{+}$(Table 1) serves as a measure of the width of the transition ${ }^{57}$. Our experiment shows that this width shrinks with increasing temperature and disappears when the temperature approaches the critical value. This intrinsic hysteretic behavior is also reproduced in the model by Bustingorry et al. ${ }^{57}$ Not only does the bulk modulus vanish according to $B \sim\left|P-P_{\mathrm{VC}+}\right|^{\alpha}$ but it also jumps discontinuously to a finite value for $p>P_{\mathrm{VC}^{-}}$. Previous experimental confirmation of this feature was seen in the data of Jeong et al. ${ }^{4}$ at room temperature. Additionally, we can now confirm that this intrinsic hysteresis disappears indeed when the system approaches the critical temperature.

While the model is based on specific assumptions that are conflicting with the properties of real materials (anisotropy and structural defects are not incorporated) the authors feel nevertheless that the features are robust enough to hold up under more general assumptions. ${ }^{57}$ A simple extension of the model would be to work with different values of bulk modulus and shear modulus on both sides of the transition.

\section{Methods}

A detailed design of the sample assembly can be found in ref. ${ }^{27}$. In summary, the $1.5 \mathrm{~mm}$ diameter and about $0.7 \mathrm{~mm}$ high polished Ce disks-polishing technique described in ref. ${ }^{58}$-were made from commercially acquired foil (Alfa Aesar, at least $99.9 \%$ purity) of $1 \mathrm{~mm}$ starting thickness. The level of impurities compares well with other previous ultrasonic experiments that used cerium disks with purity levels ranging from $98.5 \% \%^{5}, 99.53$ to $99.93^{25}$ to $99.99 \%{ }^{26}$. Impurities in our sample ( $\mathrm{Al}, \mathrm{Fe}, \mathrm{Mg}, \mathrm{Ni}, \mathrm{Si}, \mathrm{Ca}, \mathrm{La}, \mathrm{Nd}, \mathrm{Pr}$, and $\mathrm{Y}$ ) are $<0.01 \%$ each. For the ultrasonic measurements, the cylindrical sample was surrounded on the sides by crushable boron nitride (BN) and a $\mathrm{MgO}$ tube. This assembly was sitting inside another $\mathrm{BN}$ cylinder and mounted inside a graphite heater ${ }^{59}, 60$. The heater in turn was supported by another $\mathrm{MgO}$ ring pushed into amorphous boron epoxy. The outer region of the sample assembly was formed by a ring of lexan. The temperature in the sample region was determined via a previous calibration by thermocouples against power consumption ${ }^{59,60}$ with an uncertainty of $\sim 5 \mathrm{~K}$ for temperatures other than RT.

The ultrasonic pulses were transmitted to the top of the cerium disk through a cylinder of highly polished $\mathrm{Al}_{2} \mathrm{O}_{3}$. One part of the pulses was reflected back at this interface. Another disk of $\mathrm{Al}_{2} \mathrm{O}_{3}$ contacted the cerium disk on the bottom to reflect the sound waves from the bottom interface of the sample. The time difference between the two reflected pulses gave the travel time corresponding to twice the sample length. The pulse-overlap method-for details see ref. ${ }^{60}$ - was used to measure the travel time of the pulses through the sample to within nanoseconds resulting in ultrasonic velocity values with $<1 \%$ error. The frequency of the ultrasound pulses was varied between 15 and $30 \mathrm{MHz}$ since better coupling to the transverse waves was achieved at the lower frequency range, and for the longitudinal ones at $30 \mathrm{MHz}$. 
Thickness measurement of the sample to within microns in situ was provided by $\mathrm{X}$-ray radiography ${ }^{60,61}$. The X-ray beam (dimensions of $\sim 1 \mathrm{~mm} \times 1 \mathrm{~mm}$ ) produced a radiographic image on a thin scintillator crystal whose visible luminescence was focused onto a CCD camera by a microscope objective ${ }^{27}$. The length per pixel was precisely calibrated.

Energy dispersive X-ray diffraction (taken close to the outer edge of the cerium disk since the center thickness of $1.5 \mathrm{~mm}$ was opaque to the $\mathrm{X}$-rays) provided the pressure using the previously established $\operatorname{EOS}^{10}$. Most importantly, the frequent collection of X-ray diffraction data allowed the assurance that the-with temperature-increasingly reactive sample remained elemental cerium. Indeed, we did not observe any signs of reactivity during our measurements, a problem which was previously encountered in experiments much closer to the melt ${ }^{13}$.

The quasi-hydrostatic sample environment in our set-up is adequate for an accurate measurement of the transverse and longitudinal sound velocities under pressure. Experimental validation of this fact was provided by an earlier experiment on $\mathrm{SiO}_{2}$ glass that found no difference between the values for the transverse and longitudinal sound velocities obtained using the present set-up and others ${ }^{60}$. Additionally, the volume collapse transitions take place in an identical fashion to previous reports for polycrystalline cerium compressed in a diamond anvil cell. Those used either $\mathrm{NaCl}$ or helium as pressure transmitting medium ${ }^{4,10,11}$, also with no apparent difference in sample behavior. Our data begin at about $0.25 \mathrm{GPa}$ or higher since the coupling between ultrasonic waves and sample was not efficient enough at lower pressures.

All the experiments took place at the 16 BMB beam-line (HPCAT) at the Advanced Photon Source (APS) at Argonne National Lab. An important difference between our present technique and other previous ultrasonic measurements is that the sample characteristics were directly measured in situ throughout the experiment: There was no need for further assumptions and iterative schemes that would have required exact knowledge of additional thermodynamic quantities.

Data availability. The data that support the findings of this study are available from the corresponding author upon request.

Received: 27 November 2016 Accepted: 15 September 2017 Published online: 31 October 2017

\section{References}

1. Smith, J. L. \& Kmetko, E. A. Magnetism or bonding: a nearly periodic table of transition elements. J. Less Common Metals 90, 83-88 (1983).

2. Hecker, S. S. The magic of plutonium: $5 \mathrm{f}$ electrons and phase instability. Metall. Mater. Transact. A 35, 2207-2222 (2004).

3. Bridgman, P. W. The compressibility and pressure coefficient of resistance of ten elements. Proc. Am. Acad. Arts Sci. 62, 207-226 (1927).

4. Jeong, I. K. et al. The role of the lattice in the $\gamma-\alpha$ phase transition of Ce: a highpressure neutron and X-ray diffraction study. Phys. Rev. Lett. 92, 105702-105705 (2004)

5. Voronov, F. F., Vereshchagin, L. F. \& Goncharova, V. A. The effect of hydrostatic pressure on the elastic properties of cerium. Sov. Phys. Dokl. 135, $1280-1283$ (1960).

6. MacPherson, M. R., Everett, G. E., Wohlleben, D. \& Maple, M. B. Magnetic susceptibility of cerium metal under pressure. Phys. Rev. Lett. 26, 20-23 (1971).

7. Naka, T., Matsumoto, T. \& Mori, N. Magnetic states of $\alpha$ - and $\gamma$-Ce at high pressure. Physica 205, 121-126 (1995).

8. Kutsar, A. R. The $\gamma$ - $\alpha$ transformation and volume anomalies in cerium under pressure. Sov. Phys. Dokl. 24, 292-294 (1979).

9. Schiwek, A., Porsch, F. \& Holzapfel, W. B. High temperature-high pressure structural studies of cerium. High Press. Res. 22, 407-410 (2002).

10. Lipp, M. J. et al. Thermal signatures of the Kondo volume collapse in cerium. Phys. Rev. Lett. 101, 165703 (2008).

11. Decremps, F. et al. Diffusionless $\gamma-\alpha$ phase transition in polycrystalline and single-crystal cerium. Phys. Rev. Lett. 106, 065701 (2011).

12. Cadien, A. et al. First-order liquid-liquid phase transition in cerium. Phys. Rev. Lett. 110, 125503 (2013).

13. Lipp, M. J. et al. Equation of state measurements by radiography provide evidence for a liquid-liquid phase transition in cerium. J. Phys Conf. Ser. 500, 032011 (2014).

14. Schuch, A. F. \& Sturdivant, J. H. The structure of cerium at the temperature of liquid air. J. Chem. Phys. 18, 145 (1950).

15. Ashcroft, N. W. \& Mermin, N. D. Solid State Physics (Holt-Saunders International Edition, Tokyo, 1976).

16. Bradley, J. A. et al. $4 \mathrm{f}$ electron delocalization and volume collapse in praseodymium metal. Phys. Rev. B 85, 100102(R) (2012).

17. McMahan, A. K., Scalettar, R. T. \& Jarrell, M. Screening of $4 \mathrm{f}$ moments and delocalization in the compressed light rare earths. Phys. Rev. B 80, 235105 (2009).
18. Koskenmaki, D. C. \& Gschneidner, Jr K. A. in Handbook on the Physics and Chemistry of Rare Earths, Vol. 1 (eds Gschneidner, Jr K. A. \& Eyring, L.) Ch. 4 337-377 (Elsevier, Amsterdam, North Holland, 1978).

19. Johansson, B. The $\alpha-\gamma$ transition in cerium is a Mott transition. Philos. Mag. 30, 469 (1974)

20. Allen, J. W. \& Martin, R. M. Kondo volume collapse and the $\gamma-\alpha$ transition in cerium. Phys. Rev. Lett. 49, 1106 (1982).

21. Lavagna, M., Lacroix, C. \& Cyrot, M. Volume collapse in the Kondo lattice. Phys. Lett. A 90, 210 (1982).

22. Johansson, B., Abrikosov, I. A., Aldén, M., Ruban, A. V. \& Skriver, H. L. Calculated phase diagram for the $\gamma \propto$ phase transition in cerium. Phys. Rev. Lett. 74, 2335 (1995).

23. Allen, J. W. \& Liu, L. Z. $\alpha-\gamma$ transition in Ce. II. A detailed analysis of the Kondo volume-collapse model. Phys. Rev. B 46, 5047 (1992).

24. Johansson, B., Ruban, A. V. \& Abrikosov, I. A. Comment on "Thermal signatures of the Kondo volume collapse in cerium". Phys. Rev. Lett. 102, 189601 (2009).

25. Voronov, F. F., Goncharova, V. A. \& Stal'gorova, O. V. Elastic properties of cerium at pressures up to $84 \mathrm{kbar}$ and at a temperature of $293 \mathrm{~K}$. Zh. Eksp. Teor. Fiz. 76, 1351-1356 (1979).

26. Decremps, F., Antonangeli, D., Amadon, B. \& Schmerber, G. Role of the lattice in the two-step evolution of $\gamma$-cerium under pressure. Phys. Rev. B 80, 132103 (2009).

27. Lipp, M. J. et al. Strength and Debye temperature measurements of cerium across the $\gamma \rightarrow \alpha$ volume collapse: the lattice contribution. J. Phys. Condens. Matter 25, 345401 (2013).

28. Wang, Z., Bi, Y., Xu, L. \& Liu, L. Elasticity of cerium up to $4.4 \mathrm{GPa}$ by sound velocity measurements under hydrostatic pressure. Mater. Res. Express 1, 026501 (2014).

29. Huang, L. \& Chen, C. A. The lattice dynamics of $\alpha$ - and $\gamma$-Ce: a first-principles approach. J. Phys Condens. Matter 19, 476206 (2007).

30. Wang, Y. et al. Thermodynamics of the $\mathrm{Ce} \gamma-\alpha$ transition: density-functional study. Phys. Rev. B 78, 104113 (2008).

31. Tian, M. F. et al. Thermodynamics of the $\alpha-\gamma$ transition in cerium studied by an LDA + Gutzwiller method. Phys. Rev. B 91, 125148 (2015).

32. Krisch, M. et al. Phonons of the anomalous element cerium. Proc. Natl Acad. Sci. USA 108, 9342-9345 (2011).

33. Stassis, C., Gould, T., McMasters, O. D., Gschneidner, K. A. Jr \& Nicklow, R. M. Lattice and spin dynamics of $\gamma$-cerium. Phys. Rev. B 19, 5746-5753 (1979).

34. Bastide, J. P., Loriers-Susse, C., Massat, H. \& Coqblin, B. Chaleur spécifique du cérium mesurée jusqu’à 20kbar à 300K. High Temp. High Press. 10, 427-436 (1978).

35. Bergman, D. J. \& Halperin, B. I. Critical behavior of an Ising model on a cubic compressible lattice. Phys. Rev. B 13, 2145-2175 (1976).

36. Greiner, J. D., McMasters, O. D. \& Smith, J. F. Single-crystal elastic constants of $\gamma$-cerium. Scr. Metall. 14, 989 (1980).

37. Wong, J. et al. Phonon dispersions of fcc $\delta$-plutonium-gallium by inelastic $\mathrm{X}$ ray scattering. Science 301, 1078-1080 (2003).

38. Kittel, C. Introduction to Solid State Physics 4th edn, 147, Ch. 4 (Wiley, New York, 1971).

39. Boppart, H., Treindl, A., Wachter, P. \& Roth, S. First observation of a negative elastic constant in intermediate valent TmSe. Solid State Commun. 35, 483 (1980).

40. Schärer, U. \& Wachter, P. Negative elastic constants in intermediate valent $\mathrm{Sm}_{x} \mathrm{La}_{1-x} \mathrm{~S}$. Solid State Commun. 96, 497 (1995).

41. Entel, P. \& Grewe, N. Mixed valencies: structure of phase diagrams, lattice properties and the consequences of electron hole symmetry. Z. Phys. B Condens. Matter Quanta 34, 229-241 (1979).

42. Jensen, B. J., Cherne, F. J., Cooley, J. C., Zhernokletov, M. V. \& Kovalev, A. E. Shock melting of cerium. Phys. Rev. B 81, 214109 (2010).

43. Nguyen, J. H. \& Holmes, N. C. Melting of iron at the physical conditions of the Earth's core. Nature 427, 339-342 (2004).

44. Greaves, G. N., Greer, A. L., Lakes, R. S. \& Rouxel, T. Poisson's ratio and modern materials. Nat. Mater. 10, 823-837 (2011).

45. Baughman, R. H., Shacklette, J. M., Zakhidov, A. A. \& Stafström, S. Negative Poisson's ratios as a common feature of cubic metals. Nature 392, 362-365 (1998).

46. Lakes, R. Foam structures with a negative Poisson's ratio. Science 235, 1038-1040 (1987).

47. Yeganeh-Haeri, A., Weidner, D. J., Parise \& Elasticity, J. B. of $\alpha$-cristobalite: a silicon dioxide with a negative Poisson's ratio. Science 257, 650-652 (1992).

48. Suzuki, Y. et al. Temperature dependence of elastic moduli of polycrystalline $\beta$ plutonium. Phys. Rev. B 84, 064105 (2011).

49. Söderlind, P., Landa, A., Klepeis, J. E., Suzuki, Y. \& Migliori, A. Elastic properties of Pu metal and Pu-Ga alloys. Phys. Rev. B 81, 224110 (2010).

50. Rueff, J. P. et al. Probing the $\gamma$ - $\alpha$ transition in bulk Ce under pressure: a direct investigation by resonant inelastic X-Ray scattering. Phys. Rev. Lett. 96, 237403 (2006). 
51. Lipp, M. J. et al. X-ray emission spectroscopy of cerium across the $\gamma-\alpha$ volume collapse transition. Phys. Rev. Lett. 109, 195705 (2012).

52. Grimvall, G. Thermophysical Properties of Materials 85 (Elsevier, NorthHolland, 1999).

53. Amadon, B., Biermann, S., Georges, A. \& Aryasetiawan, F. The $\gamma-\alpha$ transition of cerium is entropy driven. Phys. Rev. Lett. 96, 066402 (2006).

54. Lanatà, N., Yao, Y. X., Wang, C. Z., Ho, K. M. \& Kotliar, G. Interplay of spinorbit and entropic effects in cerium. Phys. Rev. B 90, 161104(R) (2014).

55. Jarlborg, T. Role of thermal disorder for magnetism and the $\alpha-\gamma$ transition in cerium: Results from density-functional theory. Phys. Rev. B 89, 184426 (2014).

56. Moore, K. T. et al. Watching a metal collapse: examining cerium's $\gamma \leftrightarrow \alpha$ transformation using X-ray diffraction of compressed single and polycrystals. Acta Mater. 59, 6007-6016 (2011).

57. Bustingorry, S., Jagla, E. A. \& Lorenzana, J. Thermodynamics of volume collapse transitions in cerium and related compounds. Acta Mater. 53, 5183-5188 (2005)

58. Farber, D. L., Antonangeli, D., Aracne, C. M. \& Benterou, J. Preparation and characterization of single crystal samples for high pressure experiments. High Press. Res. 26, 1-10 (2006).

59. Kono, Y., Yamada, A., Wang, Y., Yu, T. \& Inoue, T. Combined ultrasonic elastic wave velocity and microtomography measurements at high pressures. Rev. Sci. Instr. 82, 023906 (2011).

60. Kono, Y. et al. Simultaneous structure and elastic wave velocity measurement of $\mathrm{SiO} 2$ glass at high pressures and high temperatures in a Paris-Edinburgh cell. Rev. Sci. Instr. 83, 033905 (2012).

61. Darling, K. L., Gwanmesia, G. D., Kung, J., Li, B. \& Liebermann, R. C. Ultrasonic measurements of the sound velocities in polycrystalline San Carlos olivine in multi-anvil, high-pressure apparatus. Phys. Earth Planet. Inter. 143-144, 19-31 (2004).

\section{Acknowledgements}

This work was performed under the auspices of the US Department of Energy by Lawrence Livermore National Laboratory under Contract No. DE-AC52-07NA27344. Portions of this work were performed at HPCAT (Sector 16), Advanced Photon Source (APS), Argonne National Laboratory. HPCAT operation is supported by DOE-NNSA under Award No. DE-NA0001974, with partial instrumentation funding by NSF. The
Advanced Photon Source is a U.S. Department of Energy (DOE) Office of Science User Facility operated for the DOE Office of Science by Argonne National Laboratory under Contract No. DE-AC02-06CH11357. Y.K., C.P., and C.K.B. acknowledge the support of DOE-BES/DMSE under Award DE-FG02-99ER45775.

\section{Author contributions}

M.J.L., H.C., and W.J.E. devised the project, M.J.L. wrote the paper with input from all authors. Y.K., C.P., and C.K.-B. developed the ultrasonic measurement capability, M.J.L. carried out the measurements with help from Zs.J., Y.K., and C.K.-B. All authors discussed the results of the manuscript.

\section{Additional information}

Competing interests: The authors declare no competing financial interests.

Reprints and permission information is available online at http://npg.nature.com/ reprintsandpermissions/

Publisher's note: Springer Nature remains neutral with regard to jurisdictional claims in published maps and institutional affiliations.

\begin{abstract}
Open Access This article is licensed under a Creative Commons Attribution 4.0 International License, which permits use, sharing, adaptation, distribution and reproduction in any medium or format, as long as you give appropriate credit to the original author(s) and the source, provide a link to the Creative Commons license, and indicate if changes were made. The images or other third party material in this article are included in the article's Creative Commons license, unless indicated otherwise in a credit line to the material. If material is not included in the article's Creative Commons license and your intended use is not permitted by statutory regulation or exceeds the permitted use, you will need to obtain permission directly from the copyright holder. To view a copy of this license, visit http://creativecommons.org/ licenses/by/4.0/
\end{abstract}

(C) The Author(s) 2017 